\title{
Managing Multi-Institutional Projects
}

Virginia Moxley, Dean and Professor, College of Human Ecology, Kansas State University

$\mathrm{T}$ The realities of current scholarly and fiscal environments sometimes make multi-institutional projects preferable to single institution undertakings. It makes sense, after all, that complicated projects are best tackled by a diverse team of experts who bring the collective resources of their employing institutions to bear on the work of the joint project. I have been engaged in major multi-institutional projects throughout the past decade and have had the opportunity to observe characteristics of successful and of ultimately unsuccessful multi-institutional collaborations.

My specific focus has been the creation and management of online graduate degrees sponsored by an alliance of major research universities. Because the projects have engaged multiple scholars employed in multiple departments at multiple universities as well as students who are admitted to graduate study at those universities, the consequences of failure are quite far reaching. Perhaps that is what propels all of us who participate in this alliance to continue our search for principles, practices, and policies that will sustain and enrich the alliance over time. The alliance that informs much of the information that follows is the Great Plains Interactive Distance Education Alliance (Great Plains IDEA). For specific information about this alliance, go to www.gpidea.org.

Some elements of multiinstitutional alliances are common to all alliances. For example, institutions are represented in the alliance by an individual and that person does not oversee all of the university entities from which support is essential if the alliance is to thrive. So, each institution will need an internal team representing the functional areas that are impacted by the alliance. The Great Plains IDEA partner institutions have found it imperative to have internal teams that include the academic deans for the colleges where the degree programs are located, the graduate dean who oversees all graduate program and student issues, the chief financial officer who can collaborate with peers at other partner institutions to reach agreement on a common price per credit hour for all students in alliance sponsored programs at all partner institutions, the participating faculty members and their department heads who manage workload issues, and a continuing education administrator who brings all of the support and regulations of the University for online delivery to the project. As Rosabeth Moss Kanter (1994) noted, the challenge of strategic alliances is that they make sense to the individual that proposes them, but they make work for others-some of whom may be actively opposed to the alliance.

In any multi-institutional project alliance, there are competing interests that must be managed. Each partner 
institution seeks to maximize institutional advantage while also contributing to the common good of the alliance because in well executed alliances, institutional interests are best served when the overall alliance flourishes. Faculty participants also have competing interests. They work on the multi-institutional project but are not employed by the alliance. Their annual merit reviews and promotion and tenure success are dependent upon departmental colleagues and administrators, whose support of alliance participation is vital to assuring that benefits rather than costs accrue to faculty as a consequence of alliance work.

Alliances are composed of a complex web of relationships. The chemistry of these relationships, according to Kanter (1994) and confirmed by my experience, is essential to their longterm viability. People with choices will only commit over time to relationships that energize and motivate them in their work - they will withdraw from relationships that deplete their energy or disadvantage them in their work.

Leadership of multi-institutional alliances is broad-based. The one essential attribute of alliance leaders is generosity. Leaders must put the interests of the alliance on a level with institutional interests. Only when there is abundant evidence that the leader behaves generously will others allow the individual to lead. Because alliances are essentially a web of relationships without much in the way of hierarchy, the maintenance of trust in leaders is an essential component in alliance viability over time.
For scholars from multiple universities to propose a joint project or program for extramural funding requires some pre-existing conditions:

- A research administration that supports the financial and reporting complexities of multi-institutional projects.

- Pre-existing relationships with the scholars from the proposed partner institutions. It is next to impossible for a group of strangers from different institutional cultures to develop a winning proposal without time to formulate a compelling common agenda, to agree on individual responsibilities, and to assign costs.

- An organizer who makes it easy for partner institutions to develop their statements of work and their budgets and who identifies and helps to collects other necessary documentation.

In a current multi-institutional project for which I serve as P.I., the timeline from release of the RFP to submission of proposals was quite brief. The proposal's success was furthered by the pre-existing relationships with the subcontract P.I.'s at all ten of the subcontracting institutions. My staff and I prepared individualized statements of work for each of the institutions based on their feedback to a query about portions of the project in which they intended to participate. We also developed and disseminated a 
formula for financial support of subcontracts and a budget template with only the bottom line (which was different for each partner institution) and indirect costs (which were at the same percentage at each partner institution) pre-computed. Institutions determined how best to allocate their project funds to accomplish their assignments. We also asked specifically for other documentation such as vitas and letters of support and provided a timeline for submission of materials. The organizer of multi-institutional proposals needs to implement a strategy that makes it easy for the partners to comply quickly and needs to implement a communications plan that assures transparency so each partner understands the basis for the distribution of funds and assignments.

Once a multi-institutional project is funded or simply undertaken, the leadership issues change from managing the transition from idea to proposal to managing the realities of working together. How can working together be hard? Let me tell you the ways.

If the alliance crosses state lines, the leaders confront different laws as well as differences in policies, practices, and institutional cultures. Often, institutional representatives think policies are laws when they are not or that prior practice is based on policy which is sometimes, but not always, the case. We have learned to ask in multiinstitutional work, whose policy or law is it? Every rule, be it a law, a policy, a practice, or a tradition comes with an address - to tweak the rule, engage the addressee. Fortunately, institutional attorneys advise on legal issues. One simply needs to ask them to investigate.

There are multiple points of disagreement about multi-institutional projects. These disagreements cannot be avoided. Ignoring conflict does not end it. When conflicts are discussed openly by individuals empowered by their employing institutions to resolve such issues, they often lead to innovative and eminently workable solutions. When they are deferred because they arouse anger or they are resolved by agreeing to a compromise that works for every institution but does not advance the project or when disagreements about administrative issues are taken on by faculty or disagreements about curriculum issues are taken on by administrators, participants become frustrated, work grinds to a stop, and commitment to the alliance is lessened.

Participants come to multiinstitutional partnerships with assumptions-most of which they have not questioned before entering into the work of alliance building. Some of these assumptions, i.e., "what is good for my institution is good for others," or "institutional practices of multiple universities are aligned" are faulty. Implicit assumptions are always present. Participants need to examine assumptions and make them explicit through developing principles to guide practice.

Communication within an alliance requires special attention to how each of the participating individuals at each of the partner institutions is best informed and best heard. The process is not unique, but its practice is rarely 
optimized. The simple mandates include:

- Conduct regular meetings either in person or via telecommunications. These meetings promote a sense of urgency about the work of the alliance and facilitate engagement by the participants. Distribute written records of the meetings promptly and expect all participants to read the written record and fulfill the assignments they assume.

- Provide information promptly to those requesting it and, when the inquiry takes on the form of a frequently asked question - provide the reply to all relevant participants.

- Make it easy for partners to do their part. Send frequent reminders about task deadlines and standards.

Alliances need to attain fiscal sustainability to endure over time. This requires the decision of one of the institutional partners to serve as fiscal agent to hold funds. In the case of academic alliances, the institutional CFO's will work to manage both the price to students and the costs to the institutions and to the alliance to assure that income covers costs. The computation of costs for new undertakings within old institutions is as much art as science. Major institutions always have sunk costs that, if assigned to the alliance, can price the program out of the market. Big bureaucracies at the center of the alliance can also doom it to fi- nancial failure as can large investments in low value outcomes.

To support the web of relationships in alliances, a managing partner institution is needed. The managing partner is compensated to provide services, to look for the next big thing for the alliance to pursue, to create web interfaces, to plan meetings, maintain a directory of participants, to exchange data securely, to promote adherence to agreements and timelines, and to communicate intrusively to help participants maintain focus on the multiuniversity project.

For academic partnerships tools such as these are needed: a secure student data management system, a student learning outcomes system, a communications system, electronic access for partners and students, and written principles, policies, and procedures. Research alliances will also require tools to make working with colleagues at partner institutions as seamless as possible.

Build redundancy in the system to accommodate abrupt changes in any partner's circumstances. The employment churn in higher education becomes a special challenge for multiinstitutional projects. One project for which I served as P.I. involved a core team of 60 individuals from 10 institutions. Over the course of the three year project, 120 individuals were part of the team due to personnel changes. The downside was the constant need to acculturate newcomers. The upside was the rapid national dissemination of the model we created as former participants moved to new institutions 
and took their alliance building experience with them.

The best alliance experiences come with serendipities. These include an expanded network of valued professional colleagues and advisors, immersion in multiple higher education cultures, rapid dissemination of technical skills because these teams work virtually most of the time, and the ability to easily capitalize on the wisdom of colleagues with similar interests.

\section{Reference}

Kanter, R.M. (1994). "Collaborative advantage: the art of alliances." Harvard Business Review, 72(4), pages 96-108. 